\title{
FERMILAB-CONF-05-245-AD \\ COMPUTATIONAL STUDY OF THE BEAM-BEAM EFFECT IN TEVATRON USING THE LIFETRAC CODE*
}

\author{
Alexander Valishev $^{\dagger}$, Yuri Alexahin, Valeri Lebedev, Fermilab, Batavia, IL 60510, USA \\ Dmitry Shatilov, BINP SB RAS, Novosibirsk, 630090, Russia
}

\begin{abstract}
Results of a comprehensive numerical study of the beam-beam effect in the Tevatron are presented including the dependence of the luminosity lifetime on the tunes, chromaticity and optics errors. These results help to understand the antiproton emittance blow-up routinely observed in the Tevatron after the beams are brought into collision. To predict a long term luminosity evolution, the diffusion rates are increased to represent long operation time ( $\sim$ day) by using a small number of simulated turns. To justify this approach, a special simulation study of interplay between nonlinear beam-beam resonances and diffusion has been conducted. A number of ways to mitigate the beam-beam effects are discussed, such as increasing bunch spacing and separation between the beams.
\end{abstract}

\section{INTRODUCTION}

In contrast to the electron-positron colliders there is no damping in hadron colliders. As the result, during the store time an effect of beam-beam interaction on the emittance growth needs to be minimized and made small relative to other diffusion mechanisms such as the intra-beam scattering (IBS), scattering on the residual gas, and diffusion due to RF phase noise. We will call these the extrinsic diffusion to distinguish from the diffusion excited by beam-beam effects. For the present Tevatron parameters the extrinsic diffusion sets the luminosity lifetime to be about 10 hours at the beginning of the store. IBS dominates both transverse and longitudinal diffusions in the case of protons while its relative effect is significantly smaller for antiprotons because of $\sim 5$ times smaller intensity.

Table 1 summarizes lifetimes for major beam parameters obtained with diffusion model [1] for a typical Tevatron store with the luminosity of $9 \times 10^{31} \mathrm{~cm}^{-2} \mathrm{~s}^{-1}$. There are many parameters in Tevatron which are beyond our control and therefore each store is different. For good stores, the beam-beam effects make comparatively small contribution to the emittance growth yielding luminosity lifetime in the range of 7-8 hours and $10-15 \%$ loss in the luminosity integral. The planned threefold increase in antiproton intensity will amplify the beam-beam effects. That, if not addressed, can cause unacceptably large background in detectors and reduce integrated luminosity. In this paper we discuss the results of numerical simulations aimed to understand major

\footnotetext{
* Work supported by the Universities Research Assoc., Inc., under contract DE-AC02-76CH03000 with the U.S. Dept. of Energy

†valishev@fnal.gov
}

factors contributing to the beam-beam interaction and possible ways of their mitigation. The details of the numerical model are outlined in [2].

\begin{tabular}{|l|c|c|}
\hline Parameter (lifetime, hour) & Protons & Antiprotons \\
\hline Luminosity & 9.6 & 9.6 \\
\hline $\begin{array}{l}\text { Transverse emittance, } \\
(d \epsilon / d t) / \epsilon \text { [hor./vert.] }\end{array}$ & $-17 /-18$ & $-52 /-46$ \\
\hline Longitudinal emittance & -8 & -26 \\
\hline Intensity & 26 & 155 \\
\hline
\end{tabular}

Table 1: Lifetimes for major beam parameters obtained with diffusion model.

\section{DIFFUSION RATE}

Under the real conditions at Tevatron the emittance growth rate is small and exact simulations of beam-beam effects would require tracking for billions of turns. That is well beyond capabilities of present computers. Fortunately, the extrinsic diffusion is large enough in comparison with beam-beam diffusion which resuls in the loss of phase correlation after about 50,000 turns.

The external noise plays important role in particle dynamics: it provides particle transport in the regions of phase space which are free from resonance islands.

To make this transport faster we can artificially increase the noise level assuming that its effect scales as noise power multiplied by number of turns. If we choose it so that the noise alone gives $10 \%$ emittance growth in $10^{6}$ turns (we use this level as the reference) then this number of turns of simulation will correspond to $\sim 5 \mathrm{~h}$ of time in the Tevatron.

To verify this approach we studied the effect of the noise level on luminosity using reconstructed optics.

Fig.1 presents results of the tune scan along the main diagonal with the reference noise level and without noise. The effect of noise on luminosity corresponds to its level with exception for the point $Q_{y}=0.575$ where it was larger due to some cooperation with strong 5th order resonances.

To study this cooperation in more detail we performed tracking at this working point with different noise levels. Fig. 2 shows the luminosity reduction in $2 \cdot 10^{6}$ turns (diamonds) and a fit made using just 3 points, with relative noise level $0.5,1$ and 2 .

The fit works fine for higher noise level, but predicts somewhat faster luminosity decay in absence of noise than actually observed in tracking. This means that there are regions in the phase space which particles cannot pass 


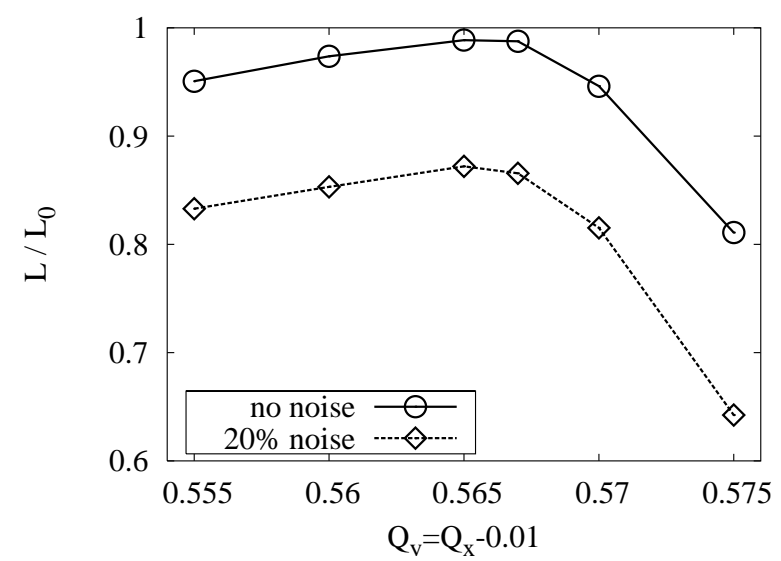

Figure 1: Ratio of luminosity after a fixed time $\left(t=2 \cdot 10^{6}\right.$ turns) to the initial luminosity vs. betatron tune. Circles $D_{\text {noise }}=0$, diamonds - the emittance change due to extrinsic diffusion after $t$ is $20 \%$.

(within the tracking time) without assistance from the external noise so that the simple rule $D_{\text {total }}=D_{\text {res }}+D_{\text {noise }}$ does not apply. However, such "blank spaces" may contain isolated resonance islands which would show up on a longer time scale with the real level of external noise. The

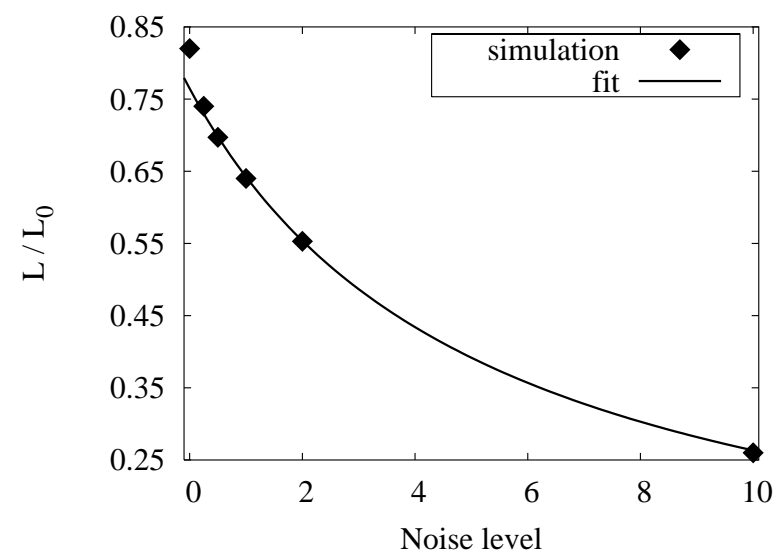

Figure 2: Ratio of luminosities vs. noise level.

applicablity of this rule at the reference noise level testifies that (with the chosen number of turns) no such "blank spaces" were left so we get more reliable predictions.

\section{RESULTS}

In this section we review some of the simulation results.

\section{Optics Errors}

In the simulations we used 3 major Tevatron optics modifications:

- "design" optics with ideal parameters of the main Interaction Points (IP), zero coupling.

- "january" optics which was in effect until March, 2004. This optics was measured in January, 2004, and had sufficient distortions in the main IPs (unequal beta's, beam waists shifted from the IP), and betatron coupling.

- "june" optics introduced in March, 2004, where the distortions were corrected.

Comparison of the three cases is shown in Fig. 3. This plot shows that modifications to the optics implemented in March, 2004, made the optics close to the design. Additional simulations revealed that the main source of particle losses was in the Parasitic Collisions (PC) nearest to the main IPs. Increasing the beams separation in these points and repairing the phase advances cured high antiproton losses.

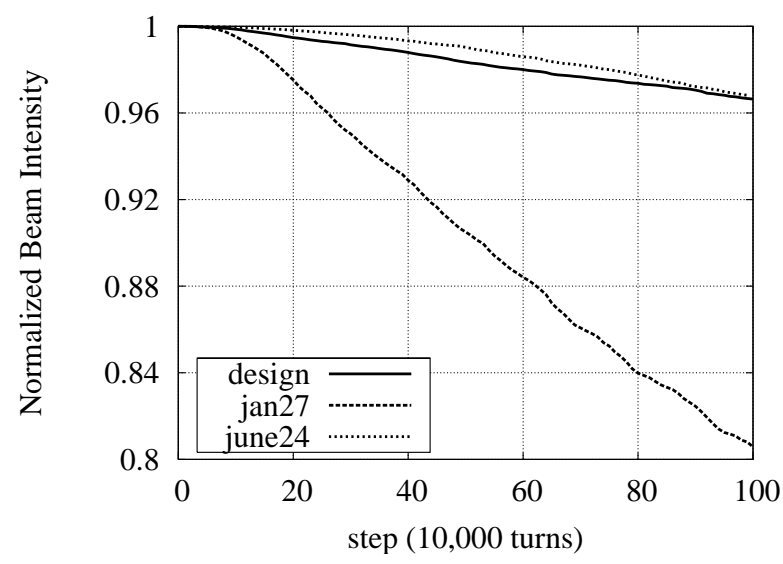

Figure 3: Intensity of bunch \#6 vs. time for different types of optics. $\xi=0.01, Q_{x}=0.57, Q_{Y}=0.56$

\section{Scallops}

Another illustration of validity of the code is simulation of scallops. Fig. 4 shows the simulated pattern of the antiproton emittance blow-up during first minute of the store. We also demonstrated that scallops can be reduced by moving the working point farther from 5 th order resonance.
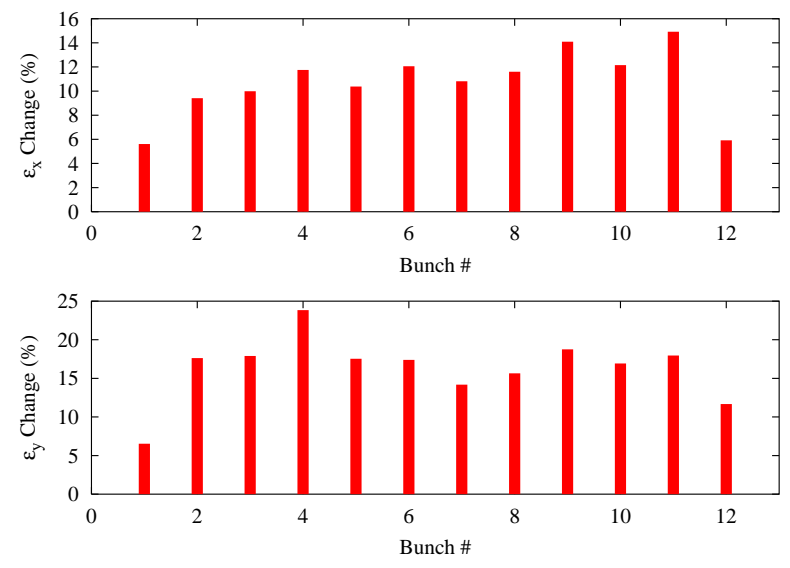

Figure 4: Change of the antiproton emittance during first $60 \mathrm{sec}$. of the store. $Q_{x}=0.575, Q_{y}=0.579, \xi=0.01$. 


\section{Chromaticity}

Reducing the betatron tune chromaticity can also be a very powerful instrument in decreasing the particle losses. Results in Figs. 5, 6 demonstrate that changing the tune chromaticity from the present 15-20 units to 5-10 units may significantly improve both the beam life time and emittance blow-up. This can give about $10 \%$ in luminosity integral.

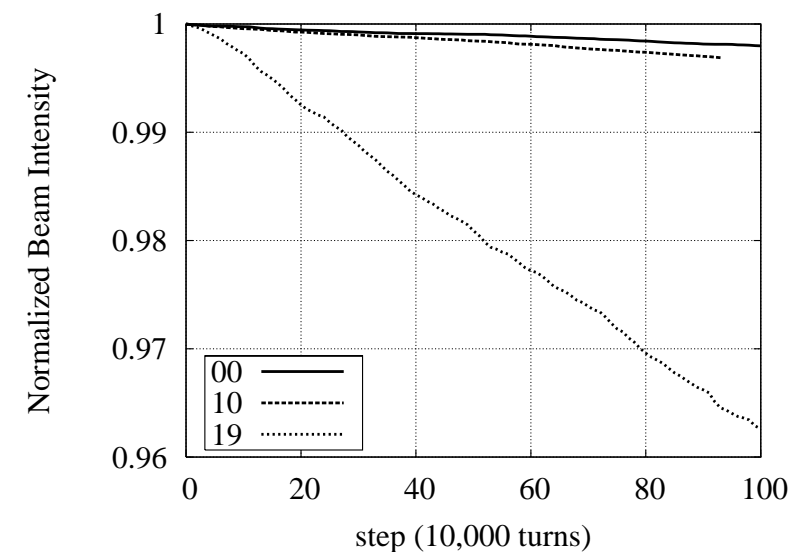

Figure 5: Evolution of the bunch intensity for various values of betatron tune chromaticity. June optics, $Q_{x}=0.58$, $Q_{y}=0.575, \xi=0.01$.

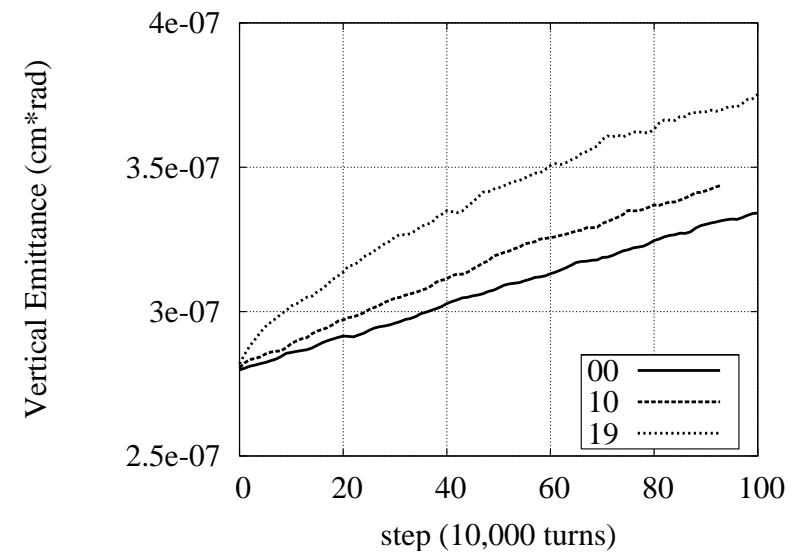

Figure 6: Vertical emittance of bunch \#6 vs. time for 3 chromaticities. Parameters are same with Fig. 5.

\section{Further $\beta^{*}$ Reduction}

An improvement which can be relatively easily implemented is the further reduction of the beta-function at the main IPs. Decreasing the $\beta^{*}$ from $35 \mathrm{~cm}$ to $28 \mathrm{~cm}$ one can gain $10 \%$ both in peak luminosity and in luminosity integral.

\section{Beams Separation, 23 Bucket Spacing}

Since Parasitic Collisions give a strong contribution to beam-beam effects, the nearest parasitic collision points dominate, one could increase separation of beams in these points thus making their effect weaker. This could be done by changing the bunch filling pattern from 3 trains of 12 bunches with 21 RF buckets between bunches to $3 \times 11$ bunches with separation of 23 RF buckets, conserving the overall intensity of antiprotons. In that case the separation in the nearest PCs is bumped from 8.2 beam sigmas to 13.6 beam sigmas. Figure 7 shows that such modification allows to increase the attainable beam-beam parameter for antiprotons from 0.01 per IP to 0.0125 without loss in efficiency.

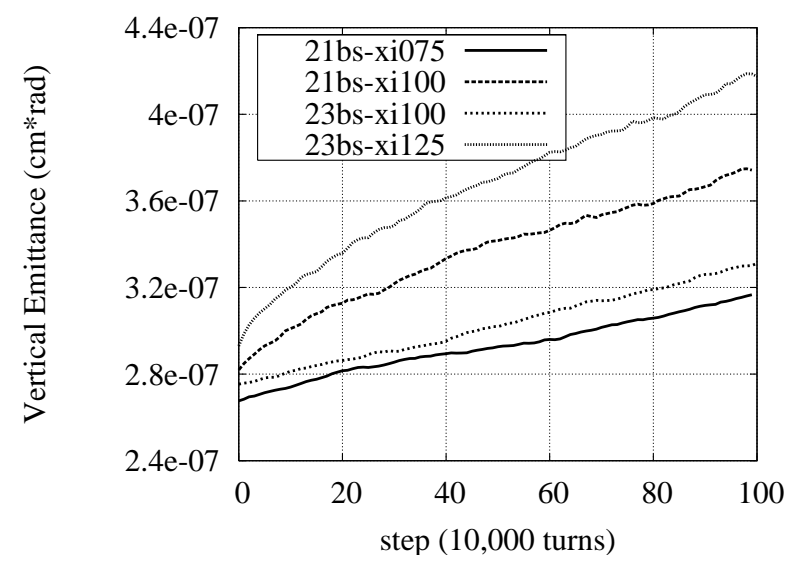

Figure 7: Vertical emittance of bunch \#6 vs. time for the 21 and 23 bucket spacing configurations and different values of beam-beam parameter.

\section{SUMMARY}

The package of programs for weak-strong beam-beam simulations for Tevatron has been developed. The program was designed to simulate the realistic machine optics and now it allows to explain some of the experimental results. A detailed study was performed to justify the increased extrinsic diffusion rate in simulations which makes it possible to simulate a relatively long period of time in the machine using limited computer resources. Based on the results of simulations, we propose several improvements in order to increase the Tevatron luminosity, including reduction of the $\beta^{*}$ value, reduction of the betatron tune chromaticity, and increasing the beams separation in the nearest (to the IP) Parasitic Collision points.

\section{ACKNOWLEDGMENTS}

The authors are grateful to V. Shiltsev and A. Tollestrup for numerous helpful discussions. We thank F. Ostiguy, M. Kriss, M. Kostin and S. Timm for help in carrying out computer simulations.

\section{REFERENCES}

[1] V. Lebedev, A. Burov, in Proceedings 33rd ICFA Workshop HB-2004, Bensheim, Germany, 2004

[2] D. Shatilov et al., TPAT084, these Proceedings 